\title{
Genotyping of the MTL loci and susceptibility to two antifungal agents of Candida glabrata clinical isolates
}

\author{
María Teresa Lavaniegos-Sobrino', Candy Y Ramírez-Zavaleta', Alfredo Ponce de León², \\ José Sifuentes-Osornio², Miriam Bobadilla-Del Valle² Andrea Rangel-Cordero², \\ Alejandro De Las Peñas ${ }^{1}$, Irene Castaño ${ }^{1 /+}$
}

División de Biología Molecular, Instituto Potosino de Investigación Científica y Tecnológica, Camino a la Presa San José 2055, Lomas 4a sección, 78216 San Luis Potosí, México ²Instituto Nacional de Ciencias Médicas y Nutrición Salvador Zubirán, México DF, México

The opportunistic fungal pathogen Candida glabrata is the second most common isolate from bloodstream infections worldwide and is naturally less susceptible to the antifungal drug fluconazole than other Candida species. C. glabrata is a haploid yeast that contains three mating-type like loci (MTL), although no sexual cycle has been described. Strains containing both types of mating information at the MTL1 locus are found in clinical isolates, but it is thought that strains containing type a information are more common. Here we investigated if a particular combination of mating type information at each MTL locus is more prevalent in clinical isolates from hospitalized patients in Mexico and if there is a correlation between mating information and resistance to fluconazole and 5-fluorocytosine. We found that while both types of information at MTL1 are equally represented in a collection of 64 clinical isolates, the vast majority of isolates contain a-type information at MTL2 and $\alpha$-type at MTL3. We also found no correlation of the particular combination of mating type information at the three MTL loci and resistance to fluconazole.

Key words: Candida glabrata - mating type loci - MTL - clinical isolate - fluconazole - 5-fluorocytosine

Candida species are successful as both commensal and opportunistic fungal pathogens, accounting for $12 \%$ of all bloodstream infections worldwide (Trick et al. 2002, Richardson 2005, Pfaller \& Diekema 2007). Candida glabrata is a haploid budding yeast that has only one known niche, the mammalian host and has recently emerged as a common opportunistic pathogen. In fact, $C$. glabrata is now the second most common Candida species isolated from bloodstream infections (Jarvis 1995, Nguyen et al. 1996) and most clinical isolates are innately less susceptible to antifungal treatment with azole compounds than other Candida species (Pfaller \& Diekema 2004, Richter et al. 2005, Messer et al. 2006, Gonzalez et al. 2008, Manzano-Gayosso et al. 2008). Phylogenetically, C. glabrata is closely related to Saccharomyces cerevisiae and close homologues of approximately $90 \%$ of $S$. cerevisiae genes can be found in the C. glabrata genome (Byrne \& Wolfe 2005).

Until recently, both Candida albicans and C. glabrata had been considered "asexual" since no mating or sexual forms had been documented. In the budding yeast $S$. cerevisiae, sexual reproduction is controlled by genes at the mating-type locus (MAT), which encodes transcription factor proteins that control the sexual cycle and possibly other

Financial support: CONACyT [MTLS (211756), CYR-Z (164574)], Fondos Sectoriales-Salud-CONACyT [APN (13927), ICN (CB-200548304)]

+ Corresponding author: icastano@ipicyt.edu.mx

Received 10 August 2008

Accepted 24 April 2009 processes (Herskowitz et al. 1992, Bahn et al. 2007). The discovery in C. albicans of various homologues of genes known to be important for mating in the related yeast $S$. cerevisiae, including a mating type-like locus (MTL), led to the discovery of a cryptic sexual phase in C. albicans (Hull \& Johnson 1999, Hull et al. 2000, Magee \& Magee 2000). This and subsequent work suggested that mating in C. albicans is regulated by unusual mechanisms that might be important for its survival in the host (Johnson 2003).

C. glabrata has also been considered an asexual organism because it has no known sexual cycle and it has a predominantly clonal population (Dodgson et al. 2005). It was recently discovered however, that $C$. glabrata, like S. cerevisiae, contains three MTL loci (MTL1, MTL2 and MTL3) (Srikantha et al. 2003), as well as the vast majority of genes involved in mating, sporulation and meiosis (Wong et al. 2003). It was reported that MTL2 and MTL3 appear to contain silent information (most frequently a and $\alpha$ information respectively) and MTL1 is thought to be the expression locus and can contain either type of information (Srikantha et al. 2003). More recently it was discovered that the information present at MTL2 is not completely silenced. C. glabrata cells however, are able to maintain cell type identity through differential regulation of expression of the $\alpha$ genes and differential splicing of the a1 gene transcript (Muller et al. 2008). The clinical isolates characterized in the previous studies identified five different classes according to the information in each $M T L$ locus with more strains containing a-type information at MTL1 although both types were found (Srikantha et al. 2003). More recently, in a worldwide collection of 198 clinical isolates, it was found that the majority of strains contained type a information at MTL1 (Brisse et al. 2009). 
In order to determine the distribution of different classes of mating type information at the $3 M T L$ loci, we determined the genotype of each of the three $M T L$ loci of 64 clinical isolates obtained from hospitalized patients at the Instituto Nacional de Ciencias Médicas y Nutrición Salvador Zubirán (INNSZ), in Mexico City, and in the Hospital Central Ignacio Morones Prieto in San Luis Potosi (HCSLP), México. In addition, several genes, such as multidrug resistance transporters and mitochondrial biogenesis genes, have been implicated in resistance to the antifungal agent fluconazole, (Rogers \& Barker 2003, Kaur et al. 2004). Because the MTL loci encode transcription factors that could regulate the expression of various genes, including those involved in antifungal drug resistance, we assessed whether there is a correlation between the information present at the MTL loci and the susceptibility to fluconazole and to 5-fluorocytosine in some of these isolates.

\section{MATERIALS AND METHODS}

Strains and oligonucleotides - Laboratory strains, clinical isolates and oligonucleotides used in this work are described in Table II. Clinical isolates obtained from INNSZ and HCSLP were previously identified as C. glabrata using Vitek identification cards (bioMerieux) and API-20 (bioMerieux).

Media - Yeast were grown on standard yeast media prepared as described previously (Sherman 1986). YPD contained $10 \mathrm{~g} / \mathrm{L}$ yeast extract (Fisher Cat. BP1422) and $20 \mathrm{~g} / \mathrm{L}$ peptone (Fisher Cat. BP1420), supplemented with $2 \%$ glucose. For plates, $2 \%$ agar was added. When required, YPD was supplemented with different concentrations of fluconazole (Diflucan-Pfizer $2 \mathrm{mg} / \mathrm{mL}$ aqueous solution) or 5-fluorocytosine (Fluka Cat. 46850).

Fluconazole and 5-fluorocytosine susceptibility assay - Twelve clinical isolates obtained from INNSZ and HCSLP were tested for susceptibility to fluconazole or 5-fluorocytosine using the broth microdilution assay with RPMI 1640 buffered with morpholinepropanesulfonic acid to $\mathrm{pH}$ 6.2. Briefly, $10^{4}$ cells were inoculated in 96-well microtiter plates containing $200 \mu \mathrm{L}$ of RPMI
1640 supplemented with $2 \%$ glucose, $0.5 \%$ ammonium sulphate and $25 \mu \mathrm{g} / \mu \mathrm{L}$ uracil. Fluconazole was added at concentrations of $8 \mu \mathrm{g} / \mu \mathrm{L}, 16 \mu \mathrm{g} / \mu \mathrm{L}, 32 \mu \mathrm{g} / \mu \mathrm{L}, 64 \mu \mathrm{g} / \mu \mathrm{L}$ and $128 \mu \mathrm{g} / \mu \mathrm{L}$. Plates were then incubated at $35^{\circ} \mathrm{C}$ and the absorbance at $\mathrm{OD}_{600}$ was read in a microplate reader every hour for $24 \mathrm{~h}$. The 5 -fluorocytosine was added at $0.05 \mu \mathrm{g} / \mu \mathrm{L}, 0.1 \mu \mathrm{g} / \mu \mathrm{L}, 0.2 \mu \mathrm{g} / \mu \mathrm{L}, 0.4 \mu \mathrm{g} / \mu \mathrm{L}, 0.8 \mu \mathrm{g} / \mu \mathrm{L}$ and $1.6 \mu \mathrm{g} / \mu \mathrm{L}$ and treated in the same way. All of these assays were carried out in triplicate and the data are the mean of the triplicates. The lowest drug concentration that reduced growth to $50 \%$ of that of the drug-free medium was defined as the $\mathrm{MIC}_{50}$ for each isolate.

Yeast genomic DNA extraction and molecular biology techniques - All yeast DNA preparations were performed according to standard protocols as described (Ausubel 2001).

PCR amplification of MTL loci - For amplification of the three MTL loci we used a set of six oligonucleotides described in Table II. After DNA extraction from each yeast strain, $1 \mu \mathrm{L}$ of the genomic DNA was used as the template for all PCR reactions using standard procedures.

The following primers and conditions were used to specifically amplify each $M T L$ locus: for MTL1 primers 146 and 149 with an annealing temperature of $56^{\circ} \mathrm{C}$, for $M T L 2$, primers 70 and 181 with an annealing temperature of $51^{\circ} \mathrm{C}$ and for $M T L 3$ primers 72 and 73 with an annealing temperature of $51^{\circ} \mathrm{C}$ (Table II). All of the PCR products were digested with the appropriate restriction enzymes to confirm the information type at each locus. All PCR products and their respective digestion products were run on $0.8 \%$ agarose gels, stained with ethidium bromide and photographed. The expected sizes of each product are described in Table I.

\section{RESULTS}

In C. glabrata, as in S. cerevisiae, most of the isolates contain two copies of one of the two types of mating information ( $a$ and $\alpha$ ), since in both organisms there are three $M T L$ loci (Fig. 1).

Clinical isolates of C. glabrata can contain either type a or type $\alpha$ at MTL1 - In order to determine whether

TABLE I

Expected fragment sizes for PCR-mating type-like locus (MTL)

\begin{tabular}{|c|c|c|c|c|c|}
\hline \multirow[b]{2}{*}{ MTL } & \multicolumn{2}{|c|}{$\begin{array}{l}\text { Fragment size expected } \\
\qquad \mathrm{kb}\end{array}$} & \multirow{2}{*}{$\begin{array}{l}\text { Digested } \\
\text { with }\end{array}$} & \multicolumn{2}{|c|}{$\begin{array}{l}\text { Fragment size expected } \\
\qquad \mathrm{kb}\end{array}$} \\
\hline & Type a & Type $\alpha$ & & Type a & Type $\alpha$ \\
\hline MTL1 & 2.302 & 2.488 & Eco RV & $\begin{array}{l}1.376 \\
0.926\end{array}$ & $\begin{array}{l}1.562 \\
0.926\end{array}$ \\
\hline MTL2 & 1.850 & 2.038 & Eco RV & $\begin{array}{l}1.406 \\
0.444\end{array}$ & $\begin{array}{l}1.594 \\
0.444\end{array}$ \\
\hline MTL3 & 2.339 & 2.530 & $A c c 65 \mathrm{I}$ & $\begin{array}{l}1.370 \\
0.619 \\
0.350\end{array}$ & $\begin{array}{l}2.180 \\
0.350\end{array}$ \\
\hline
\end{tabular}


TABLE II

Strains and oligonucleotides used in this study

\begin{tabular}{|c|c|c|c|}
\hline Strain & Parent & \multicolumn{2}{|c|}{ Relevant genotype/reference } \\
\hline BG14 & BG1, clinical isolate & \multicolumn{2}{|c|}{$\begin{array}{c}\text { ura3 } 3(-85+932):: \operatorname{Tn} 903 \mathrm{Neo}^{\mathrm{R}}, \text { MTL1a } \\
\text { (Cormack \& Falkow 1999) }\end{array}$} \\
\hline CBS138 & Clinical isolate & \multicolumn{2}{|c|}{ wild-type, $M T L 1 \alpha($ Genolevures 2004) } \\
\hline MC1 through 64 & Clinical isolates & \multicolumn{2}{|c|}{ INNSZ and HCSLP } \\
\hline \multicolumn{4}{|l|}{ Oligonucleotides } \\
\hline Primer & Seque & & Restriction site \\
\hline 70 & СТАТTСТААТTG & & \\
\hline 72 & GAAGTTTAAA & & \\
\hline 73 & GAATTGTCACA & & \\
\hline 146 & CCGGTACCTTGTGCAGCTG & ACCATC & $K p n \mathrm{I}, B s g \mathrm{I}$ \\
\hline 149 & CCGAGCTCTTGTGCAGGCG' & ГATGATG & Sac I, Bsg I \\
\hline 181 & CCTTAGTGATT & & \\
\hline 197 & GGCTGTAGTTC & & \\
\hline 199 & GACAGTCGCTCC & & \\
\hline
\end{tabular}

a: sequence is from 5'-3'; HCSLP: Hospital Central Ignacio Morones Prieto, San Luis Potosí, Mexico; INNSZ: Instituto Nacional de Ciencias Médicas y la Nutrición Salvador Zubirán, Mexico City, Mexico.

any particular arrangement of the information present at each of the MTL loci is more common in a collection of 64 clinical isolates obtained from hospitalized patients (Table II), we designed three pairs of oligonucleotides (Table II) that specifically amplify each of the three MTL loci. Fig. 1 A, B show a schematic representation of the configuration of the MTL loci in C. glabrata, the position where each primer set anneals and the expected size of the fragment amplified for each locus for each of the two possible information types. We PCR amplified the MTL1 locus from each of the 64 clinical isolates, as well as two laboratory strains that served as controls: BG14 (MTL1a genotype) and CBS138 (MTL1 $\alpha$ genotype) (Table II). Fig. 2A shows the bands amplified from each of 10 representative clinical isolates and the two control reference strains. In this example it can be seen that the $\alpha$ information produces a larger band that is easily distinguishable by gel electrophoresis. All of the PCR products were digested with appropriate restriction enzymes to generate smaller fragments specific for each type of information to unambiguously determine the genotype present at each locus (data not shown, see Materials and Methods). We found that both types of information at MTL1 were almost equally represented (with possibly a small overrepresentation of the $\alpha$ type). Among the 64 clinical isolates analyzed, $42.2 \%$ were MTLla and $54.7 \%$ were MTL1 $\alpha$ (Table III). Two isolates (MC28 and MC54) could not be typed at this locus since we could not amplify any band from this locus using the primers described in Table II. However, we were able to amplify both junction fragments from both of these isolates using primers 146 and 197 (annealing within EMG1 and $\alpha 1$ respectively) and primers 149 and 199 (annealing within $B U D 5$ and $\alpha 2$ respectively), suggesting the presence of a large insertion between $\alpha 1$ and $\alpha 2$ or some other rearrangement (data not shown). This also suggests that these isolates originally contained type $\alpha$ information at MTL1. It is interesting to note that in one of these two isolates, MTL3 could also not be amplified, although a band corresponding to MTL2a was amplified (MC28) (Table III). The other isolate contained type a and type $\alpha$ information for MTL2 and MTL3 respectively (MC54) (Table III). We are currently investigating the nature of the rearrangement or insertion in MTL1 of these isolates.

Most clinical isolates contain type a information at MTL2 and $\alpha$ at MTL3 - Table IV shows the classification of all the clinical isolates according to the particular combination of information contained in each of the three MTL loci. Five different classes of $C$. glabrata strains have been reported previously based on the mating type information in each of the three MTL loci of 38 clinical isolates (Srikantha et al. 2003), although theoretically, there are eight possible combinations as indicated in Table IV. In this classification we only included the 62 clinical isolates from which all three MTL loci could be amplified. As can be seen in Tables III and IV, the majority of the clinical isolates $(60 / 62$ or $96.8 \%)$ contain a information at MTL2 and $\alpha$ information at MTL3 (61/62 or $98.4 \%$ ). There were only two isolates with $\alpha$ information in MTL2 and only one with a information in MTL3.

There is no correlation between the particular information at the MTL loci and susceptibility to fluconazole - In order to determine whether there was any correlation between the different classes of clinical isolates of C. glabrata and the sensitivity of these strains to fluconazole, we performed a susceptibility assay to this drug using a broth microdilution assay. Consistent with what 
A

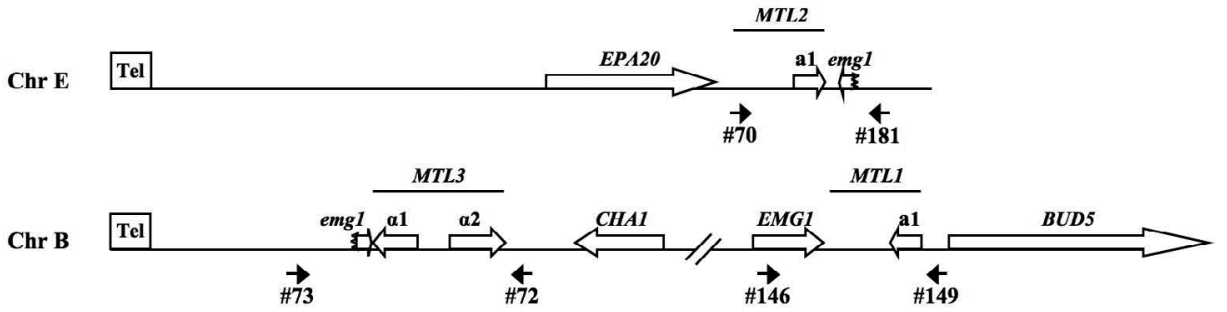

B

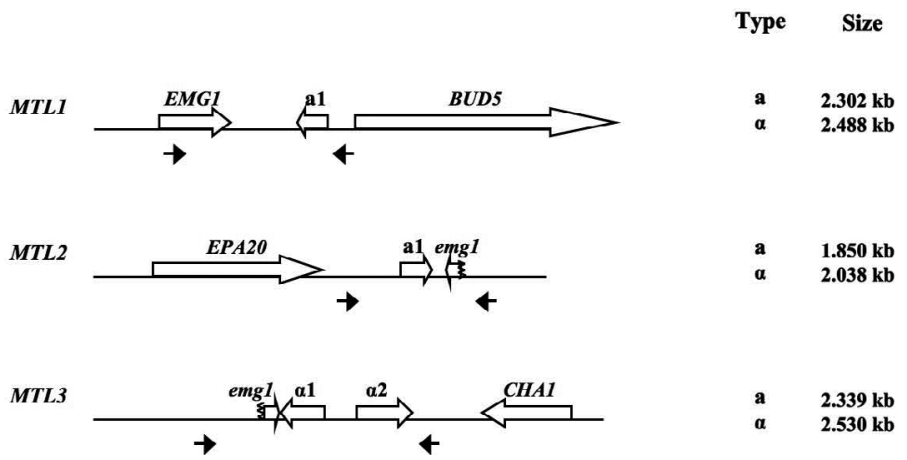

Fig. 1: schematic representation of the mating type-like locus (MTL) of Candida glabrata. A: map of the MTL1, MTL2 and MTL3 loci. The genes flanking these loci are represented by empty arrows indicating the direction of transcription. MTL2 and MTL3 are located close to the left telomeres of chromosomes E and B, respectively. The positions of each pair of oligonucleotides used for the amplification of the MTL loci are indicated. Only the EMG1 gene at the MTL1 locus is complete, the other two loci contain only partial duplications of the C-terminal region of this gene (emgl), shown as truncated arrows; B: linear map of the MTL loci indicating the position of the primer pairs used to amplify each locus. The sizes of the specific PCR products expected for each MTL locus are indicated and differs depending on whether the information contained is either type a or type $\alpha$.

has been previously reported (Kaur et al. 2004, Pfaller \& Diekema 2004, Messer et al. 2006), we found that 10 of the 12 strains tested, grew in RPMI 1640 supplemented with $8 \mu \mathrm{g} / \mathrm{mL}$ of fluconazole (Table VI). This correlates very well with the growth breakpoint on fluconazole plates previously determined for laboratory strains BG14 and CBS138 (Kaur et al. 2004). Analysis of the drug sensitivity data indicates that there is no clear correlation between the particular arrangement of information at the $M T L$ loci and the level of resistance to fluconazole. For example, MC3, MC4, MC6 and MC8 (MTL1a, MTL2a and $M T L 3 \alpha$ ) are resistant to $32-128 \mu \mathrm{g} / \mathrm{mL}$ of fluconazole, but the control strain CBS138 and MC1 and MC5, which are identical at the $M T L$ loci, are susceptible to $\geq$ $8 \mu \mathrm{g} / \mathrm{mL}$ of fluconazole (Tables V, VI). Similarly, MC7, MC17 and MC44 (MTLla, MTL2a and MTL3a) are resistant to fluconazole at $128 \mu \mathrm{g} / \mathrm{mL}$, but both the control strain BG14 and MC2 (with the same genotype at the MTL loci) are sensitive to $16-32 \mu \mathrm{g} / \mathrm{mL}$ (Tables V, VI). Furthermore, clinical isolates MC14 (MTLla, MTL2a MTL3a) and MC16 (MTL1a, MTL2 $\alpha$ MTL3 $\alpha$ ) which are genotypically different from the previous two groups are both resistant to high levels of fluconazole.

We also determined the susceptibility to 5-fluorocytosine using the microdilution assay in RPMI 1640 with increasing concentrations of 5-fluorocytosine ranging from 0.05-1.6 $\mu \mathrm{g} / \mu \mathrm{L}$. As shown in Table VI, most clini- cal isolates are sensitive to 5-fluorocytosine and some of them could not grow in the presence of the lowest concentration of 5-fluorocytosine used $(0.05 \mu \mathrm{g} / \mu \mathrm{L})$. However, there were three clinical isolates that could grow to $50 \%$ (or more) of the $\mathrm{OD}_{600}$ attained in the drug-free media, when 5-fluorocytosine was added to $1.6 \mu \mathrm{g} / \mu \mathrm{L}$ (MC3, $\mathrm{MC} 17$ and MC44). Of these 5-fluorocytosine resistant isolates, one of them contains type $\alpha$ information (MC3), and the other two, type a information at MTL1 (MC17 and MC44). We are currently determining the 5-fluorocytosine susceptibility of all 64 clinical isolates.

\section{DISCUSSION}

The fungal pathogen C. glabrata has no known sexual cycle even though it contains close homologues of the genes that control the mating type in S. cerevisiae (MTL) as well as the vast majority of the genes involved in mating and meiosis. The genes encoded at the MTL loci are transcription factors that control the mating information of the cell and also probably other processes, some of which could be important for pathogenesis (Bahn et al. 2007). It is known that many fungal pathogens limit their sexual reproduction, but still retain the capacity to reproduce sexually under certain laboratory and in vivo conditions (Heitman 2006).

To begin addressing whether $C$. glabrata could have a sexual phase and if the transcription factors encoded in 


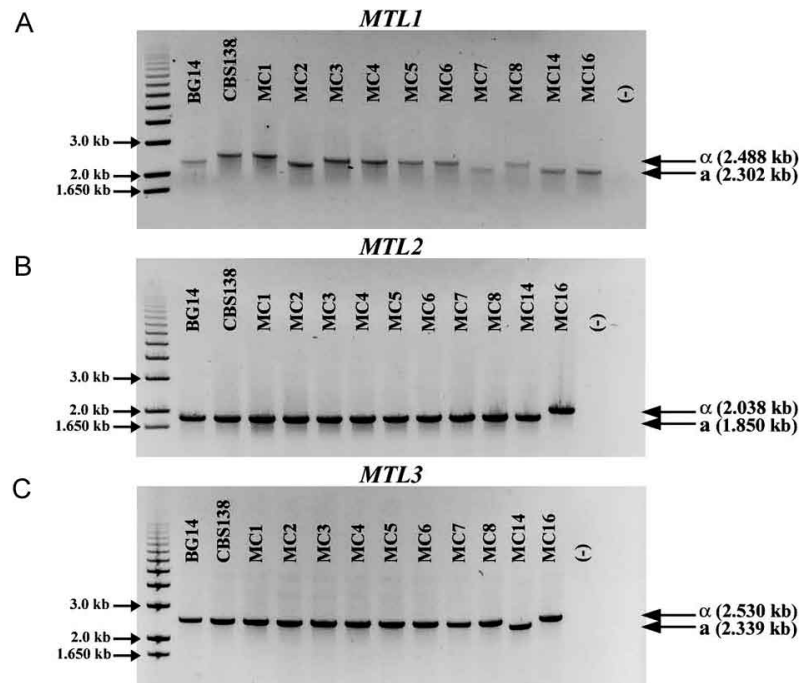

Fig. 2: genotypification of the mating type-like locus $(M T L)$ in Candida glabrata. A: agarose gel of the amplification products of MTL1 locus using the specific primers \# 146 and \#149 (Fig. 1). The gel shows only 10 clinical isolates (MC strains) and the two reference strains BG14 (MTL1a) and CBS138 (MTL1 $\alpha$ ). The expected size of the PCR products for information type a or type $\alpha$ is indicated; B: agarose gel of the amplification products of MTL2 locus of the same clinical isolates used in A. The specific primers are \# 70 and \# 181 (Fig. 1). The expected size of the PCR products for information type a or type $\alpha$ is indicated; $\mathrm{C}$ : agarose gel of the amplification products of $M T L 3$ locus of the same clinical isolates used in A. The specific primers are \# 72 and \# 73 (Fig. 1). The expected size of the PCR products for information type a or type $\alpha$ is indicated.

the MTL loci could regulate the expression of genes involved in the innate resistance of C. glabrata to antifungal drugs, we set out to analyze the information encoded in each of the three MTL loci of 64 clinical isolates obtained from hospitalized patients in Mexico.

In a previous study with a collection of 38 clinical isolates it was determined that strains with type a information at MTL1 are more common than those containing type $\alpha$ information at this locus (Srikantha et al. 2003, Muller et al. 2008). In a more recent study, it was found that in a large world-wide collection of 162 clinical isolates, the MTLla genotype was much more frequent than the MTLl $\alpha$ genotype ( $80 \%$ vs. $20 \%$ ) and that the two alleles were unevenly distributed (Brisse et al. 2009). However, in our sample of 62 clinical isolates from different geographical sites in Mexico, we found that both types of information are almost equally represented and that the $\alpha$ information might even be more prevalent (Table III), indicating that among the clinical isolates from hospitalized patients in Mexico, both genotypes are common. It is possible that this difference is due to the smaller sample that was characterized in this work or that the isolates obtained in this study are biased in some unknown way. We are currently analyzing whether there is a correlation between the clinical data of the patients (site of colonization, treatment etc.) and a specific mating type information.
TABLE III

Determination of the genotype at the MTL1, MTL2 and MTL3 loci of 64 clinical isolates

\begin{tabular}{|c|c|c|c|}
\hline \multirow[b]{2}{*}{ Information type } & \multicolumn{3}{|c|}{$\begin{array}{l}\text { Clinical isolates containing } \\
\text { each mating information } \\
\qquad \%(\mathrm{n})^{a}\end{array}$} \\
\hline & MTL1 & MTL2 & MTL3 \\
\hline $\mathrm{a}$ & $42.2(27 / 64)$ & $96.9(62 / 64)$ & $1.6(1 / 64)$ \\
\hline$\alpha$ & $54.7(35 / 64)$ & $3.1(2 / 64)$ & $96.8(62 / 64)$ \\
\hline not identified $^{b}$ & $3.1(2 / 64)$ & $0(0 / 64)$ & $1.6(1 / 64)$ \\
\hline
\end{tabular}

$a$ : numbers show the percentage of clinical isolates that contain the specified mating information at the indicated locus. The numbers in parenthesis indicate the number of isolates containing the indicated information from the total of 64 clinical isolates tested; $b$ : two clinical isolates did not give amplification bands with the oligonucleotides used to amplify mating type-like locus (MTL1). Of these, one (MC54) gave amplification bands for MTL2 and MTL3 (a and $\alpha$ information, respectively). The second (MC28) only MTL2 could be amplified (a information), but not MTL3.

\section{TABLE IV}

Classification and percentage of clinical isolates containing different combinations of mating type information at each of the three mating type-like locus (MTL)

\begin{tabular}{lcccc}
\hline Class & MTL1 & MTL2 & MTL3 & $\%\left(\mathrm{n}^{a}\right)$ \\
\hline $1^{b}$ & $\mathrm{a}$ & $\mathrm{a}$ & $\mathrm{a}$ & $1.6(1 / 62)$ \\
$2^{c}$ & $\mathrm{a}$ & $\mathrm{a}$ & $\alpha$ & $38.7(24 / 62)$ \\
3 & $\mathrm{a}$ & $\alpha$ & $\mathrm{a}$ & $0(0 / 62)$ \\
$4^{d}$ & $\mathrm{a}$ & $\alpha$ & $\alpha$ & $3.2(2 / 62)$ \\
$5^{e}$ & $\alpha$ & $\alpha$ & $\alpha$ & $0(0 / 62)$ \\
6 & $\alpha$ & $\alpha$ & $\mathrm{a}$ & $0(0 / 62)$ \\
$7^{f}$ & $\alpha$ & $\mathrm{a}$ & $\alpha$ & $56.5(35 / 62)$ \\
8 & $\alpha$ & $\mathrm{a}$ & $\mathrm{a}$ & $0(0 / 62)$ \\
\hline
\end{tabular}

$a$ : number of isolates containing the indicated information. Only 62 isolates were included and two could not be classified because there were no amplified bands with one or more pairs of primers; $b$ : corresponds to class V (Srikantha et al. 2003); $c$ : corresponds to class I (Srikantha et al. 2003); $d$ : corresponds to class III (Srikantha et al. 2003); $e$ : corresponds to Class IV (Srikantha et al. 2003); $f$ : corresponds to Class II (Srikantha et al. 2003).

In $C$. glabrata, the orthologues of $S$. cerevisiae $H M R$ and $H M L$ are MTL2 and MTL3 respectively. In both organisms, in the vast majority of the strains tested, the information present at these two loci is MTL2a and $M T L 3 \alpha$. These results corroborate and expand the previous characterization of these loci in 38 clinical isolates (Srikantha et al. 2003). We found one isolate (class 1) that contained only type a information at the three MTL loci, whereas Srikantha et al. (2003) found one isolate 
TABLE V

Susceptibility to fluconazole of clinical isolates

\begin{tabular}{|c|c|c|c|c|c|c|}
\hline \multirow[b]{2}{*}{ Clinical isolate } & \multicolumn{3}{|c|}{ Mating type information } & \multirow{2}{*}{$\begin{array}{c}\text { Isolates tested } \\
\mathrm{n}\end{array}$} & \multicolumn{2}{|c|}{$\begin{array}{c}\text { Fluconazole }^{a} \\
\%\end{array}$} \\
\hline & MTL1 & MTL2 & MTL3 & & Resistant $^{b}$ & Sensitive $^{c}$ \\
\hline $\begin{array}{l}\text { MC: } 2,7,17,44 \\
\text { (class 2) }\end{array}$ & a & a & $\alpha$ & 4 & $\begin{array}{c}75(3 / 4) \\
(\mathrm{MC}: 7,17,44)\end{array}$ & $\begin{array}{c}25(1 / 4) \\
(\mathrm{MC} 2)\end{array}$ \\
\hline $\begin{array}{l}\text { MC: } 1,3,4,5,6,8 \\
\text { (class 7) }\end{array}$ & $\alpha$ & a & $\alpha$ & 6 & $\begin{array}{c}66.6(4 / 6) \\
(\mathrm{MC}: 3,4,6,8)\end{array}$ & $\begin{array}{c}33(3 / 6) \\
(\mathrm{MC}: 1,5)\end{array}$ \\
\hline $\begin{array}{l}\text { Control BG14 } \\
\text { (class 2) }\end{array}$ & a & a & $\alpha$ & $\begin{array}{l}\text { Reference } \\
\text { strain }^{d}\end{array}$ & & Sensitive $^{e}$ \\
\hline $\begin{array}{l}\text { Control CBS138 } \\
\text { (class } 7 \text { ) }\end{array}$ & $\alpha$ & a & $\alpha$ & $\begin{array}{l}\text { Reference } \\
\text { strain }^{d}\end{array}$ & & Sensitive \\
\hline $\begin{array}{l}\text { MC14 } \\
\text { (class 1) }\end{array}$ & $\mathrm{a}$ & a & a & 1 & Resistant & \\
\hline $\begin{array}{l}\text { MC16 } \\
\text { (class 4) }\end{array}$ & a & $\alpha$ & $\alpha$ & 1 & Resistant & \\
\hline
\end{tabular}

$a$ : percentage of clinical isolates that show resistance or sensitivity to fluconazole (see footnotes $b$ and $c$ ). The numbers in parenthesis indicate the number of isolates showing resistance or sensitivity to fluconazole from a total of 12 clinical isolates tested; $b$ : resistant means that cells can grow in RPMI 1640 with $\geq 32 \mu \mathrm{g} / \mathrm{mL}$ fluconazole to at least $50 \%$ the OD 600 attained in RPMI 1640 without drug. MC3, MC8 can grow with $32 \mu \mathrm{g} / \mathrm{mL}$ of fluconazole and isolates MC4, MC6, MC7, MC14, MC16, MC17 and MC44 with $128 \mu \mathrm{g} / \mathrm{mL}$ of fluconazole; $c$ : sensitive means that cells grow in RPMI 1640 with up to $8 \mu \mathrm{g} / \mathrm{mL}$ of fluconazole, but growth is inhibited (to less than $50 \%$ of the growth attained without fluconazole) at $\geq 16 \mu \mathrm{g} / \mathrm{mL}$ of the drug (this marks a breakpoint); $d$ : control laboratory strains BG14 and CBS138 (Cormack \& Falkow 1999, Genolevures 2004); $e$ : control strain BG14 shows a sensitive-dose dependent phenotype with fluconazole concentrations from $16 \mu \mathrm{g} / \mathrm{mL}$ to $32 \mu \mathrm{g} / \mathrm{mL}$ in RPMI-1640 liquid medium. The breakpoint of this strain on YPD plates is observed at $16 \mu \mathrm{g} / \mathrm{mL}$ (Kaur et al. 2004).

TABLE VI

Susceptibility to fluconazole and 5-fluorocytosine of clinical isolates

\begin{tabular}{|c|c|c|c|c|}
\hline \multirow[b]{2}{*}{ Clinical isolate } & \multicolumn{2}{|c|}{$\mathrm{MIC}_{50}{ }^{a}$} & \multirow[b]{2}{*}{$\begin{array}{c}\text { Site of } \\
\text { colonization }^{b}\end{array}$} & \multirow[b]{2}{*}{$\begin{array}{l}\text { Geographical origin/ } \\
\text { city of isolation }^{c}\end{array}$} \\
\hline & Fluconazole $(\mu \mathrm{g} / \mathrm{mL})$ & $\begin{array}{l}\text { 5-fluorocytosine } \\
\qquad(\mu \mathrm{g} / \mathrm{mL})\end{array}$ & & \\
\hline Control BG14 & 32 & 0.4 & vaginal mucosa & \\
\hline Control CBS138 & 8 & 0.1 & human feces & \\
\hline $\mathrm{MC1}$ & $<8$ & $<0.05$ & endodontia & San Luis Potosi \\
\hline MC2 & 16 & 0.1 & urine & San Luis Potosi \\
\hline MC3 & 32 & 1.6 & urine & San Luis Potosi \\
\hline MC4 & $>128$ & 0.4 & urine & San Luis Potosi \\
\hline MC5 & 8 & 0.1 & urine & San Luis Potosi \\
\hline MC6 & $>128$ & $<0.05$ & urine & San Luis Potosi \\
\hline MC7 & $>128$ & 0.8 & urine & San Luis Potosi \\
\hline MC8 & 32 & $<0.05$ & vaginal mucosa & León \\
\hline MC14 & $>128$ & 0.4 & blood & México City \\
\hline $\mathrm{MC} 16$ & $>128$ & 0.1 & blood & México City \\
\hline MC17 & $>128$ & 1.6 & blood & México City \\
\hline MC44 & $>128$ & 1.6 & blood & México City \\
\hline
\end{tabular}

$a$ : $\mathrm{MIC}_{50}$ was defined as the minimal concentration of fluconazole or 5-fluorocytosine that resulted in a growth decrease of at least 50\% compared to that in the drug-free RPMI-1640 media; $b$ : body sites from where the clinical isolates were obtained from hospitalized patients. Control strains have been previously described (reference is in Table II); $c$ : all clinical isolates from this study (MC) were obtained in Mexico from different cities/states and hospitals. 
that contained type $\alpha$ information at all three loci (class 5) (Table IV). Classes 3 and 6, in which both loci contain the opposite information (MTL2 $\alpha$ and MTL3a), have not yet been identified. Class 8 , in which information at both MTL2 and MTL3 is type a and type $\alpha$ at MTL1, has also not yet been identified, although this seems likely to exist since the same configuration at MTL2 and MTL3 but with type a information also at MTL1 was identified (class 1, MC14) (Table IV).

We also asked whether the information present at the MTL loci could have an effect on the susceptibility of the strains to the antifungal drugs fluconazole and 5 -fluorocytosine. All but one of the 12 strains that we tested for fluconazole sensitivity, showed resistance to $8 \mu \mathrm{g} / \mathrm{mL}$ of the drug and these data further confirm the fact that C. glabrata is innately resistant to azole compounds compared to other Candida species. We found nine isolates that were resistant to high concentrations of fluconazole and three that were sensitive to the drug, but we could find no clear correlation between the genotype at the MTL loci and the level of resistance (Table V). We conclude, therefore, that in the sample we have analyzed, the information present at the mating loci does not have an impact on the level of susceptibility to the antifungal drug fluconazole. With the antifungal drug 5-fluorocytosine, we found that only three of the 12 clinical isolates tested were resistant to this drug and these were also resistant to high levels of fluconazole. In the case of the 5-fluorocytosine resistant isolates, two of the three have type a information at MTL1 and the other contains $\alpha$ information. Therefore, with the small sample we have analyzed so far, there seems to be no direct correlation between information at the MTL loci and resistance to 5-fluorocytosine. It will therefore be interesting to investigate whether there is a correlation between the treatment the patients received (or sites of colonization from where these strains were collected) and the resistance level to fluconazole and 5-fluorocytosine. It will also be important to determine whether some of the classes outlined in Table IV are more fit for colonizing certain sites or tissues in the mammalian host using murine models of infection.

\section{REFERENCES}

Ausubel F, Brent R, Kingston RE, Moore dD, Seidman JG, Smith JA, Struhl K 2001. Current protocols in molecular biology, Wiley \& Sons, New York, 670 pp.

Bahn YS, Xue CY, Idnurm A, Rutherford JC, Heitman J, Cardenas ME 2007. Sensing the environment: lessons from fungi. Nat Rev Microbiol 5: 57-69.

Brisse S, Pannier C, Angoulvant A, de Meeus T, Diancourt L, Faure O, Muller H, Peman J, Viviani MA, Grillot R, Dujon B, Fairhead C, Hennequin C 2009. Uneven distribution of mating types among genotypes of Candida glabrata isolates from clinical samples. Eukaryot Cell 8: 287-295.

Byrne KP, Wolfe KH 2005. The yeast gene order browser: combining curated homology and syntenic context reveals gene fate in polyploid species. Genome Res 15: 1456-1461.

Cormack BP, Falkow S 1999. Efficient homologous and illegitimate recombination in the opportunistic yeast pathogen Candida glabrata. Genetics 151: 979-987.
Dodgson AR, Pujol C, Pfaller MA, Denning DW, Soll DR 2005. Evidence for recombination in Candida glabrata. Fungal Genet Biol 42: 233-243.

Genolevures - French Bioinformatics Platforms Networks [Bordeaux Bioinformatics Center] 2004. Available from: http://www.genolevures.org/elt/CAGL/CAGL.

Gonzalez GM, Elizondo M, Ayala J 2008. Trends in species distribution and susceptibility to seven antifungal agents of bloodstream isolates of Candida in Monterrey, Mexico. Results of a 3-Year (2004-2007). Surveillance Study. J Clin Microbiol 46: 2902-2905.

Heitman J 2006. Sexual reproduction and the evolution of microbial pathogens. Curr Biol 16: R711-R725.

Herskowitz J, Rine J, Strathern J 1992. Mating type determination and mating-type interconversion in Saccharomyces cerevisiae, Cold Spring Harbor Laboratory Press, New York, 810 pp.

Hull CM, Johnson AD 1999. Identification of a mating type-like locus in the asexual pathogenic yeast Candida albicans. Science 285: 1271-1275.

Hull CM, Raisner RM, Johnson AD 2000. Evidence for mating of the "asexual" yeast Candida albicans in a mammalian host. Science 289: 307-310.

Jarvis WR 1995. Epidemiology of nosocomial fungal infections with emphasis on Candida species. Clin Infect Dis 20: 1526-1530.

Johnson A 2003. The biology of mating in Candida albicans. Nat Rev Microbiol 1: 106-116.

Kaur R, Castano I, Cormack BP 2004. Functional genomic analysis of fluconazole susceptibility in the pathogenic yeast Candida glabrata: roles of calcium signaling and mitochondria. Antimicrob Agents Chemother 48: 1600-1613.

Magee BB, Magee PT 2000. Induction of mating in Candida albicans by construction of MTLa and MTLalpha strains. Science 289: 310-313.

Manzano-Gayosso P, Mendez-Tovar LJ, Hernandez-Hernandez F, Lopez-Martinez R 2008. Antifungal resistance: an emerging problem in Mexico. Gac Med Mex 144: 23-26.

Messer SA, Jones RN, Fritsche TR 2006. International surveillance of Candida spp. and Aspergillus spp.: report from the SENTRY Antimicrobial Surveillance Program (2003). J Clin Microbiol 44: $1782-1787$.

Muller H, Hennequin C, Gallaud J, Dujon B, Fairhead C 2008. The asexual yeast Candida glabrata maintains distinct a and alpha haploid mating types. Eukaryot Cell 7: 848-858.

Nguyen MH, Peacock JE Jr, Morris AJ, Tanner DC, Nguyen ML, Snydman DR, Wagener MM, Rinaldi MG, Yu VL 1996. The changing face of candidemia: emergence of non-Candida albicans species and antifungal resistance. Am J Med 100: 617-623.

Pfaller MA, Diekema DJ 2004. Twelve years of fluconazole in clinical practice: global trends in species distribution and fluconazole susceptibility of bloodstream isolates of Candida. Clin Microbiol Infect 10 (Suppl. 1): 11-23.

Pfaller MA, Diekema DJ 2007. Epidemiology of invasive candidiasis: a persistent public health problem. Clin Microbiol Rev 20: $133-163$.

Richardson MD 2005. Changing patterns and trends in systemic fungal infections. J Antimicrob Chemother 56 (Suppl. 1): i5-i11.

Richter SS, Galask RP, Messer SA, Hollis RJ, Diekema DJ, Pfaller MA 2005. Antifungal susceptibilities of Candida species causing vulvovaginitis and epidemiology of recurrent cases. J Clin Microbiol 43: 2155-2162. 
Rogers PD, Barker KS 2003. Genome-wide expression profile analysis reveals coordinately regulated genes associated with stepwise acquisition of azole resistance in Candida albicans clinical isolates. Antimicrobial Agents Chemother 47: 1220-1227.

Sherman F, Fink GR, Hicks JB 1986. Methods in yeast genetics, Cold Spring Harbor Laboratory Press, New York, 186 pp.

Srikantha T, Lachke SA, Soll DR 2003. Three mating type-like loci in Candida glabrata. Eukaryot Cell 2: 328-340.
Trick WE, Fridkin SK, Edwards JR, Hajjeh RA, Gayne RP 2002. Secular trend of hospital-acquired candidemia among intensive care unit patients in the United States during 1989-1999. Clin Infect Dis 35: 627-630.

Wong S, Fares MA, Zimmermann W, Butler G, Wolfe KH 2003. Evidence from comparative genomics for a complete sexual cycle in the 'asexual' pathogenic yeast Candida glabrata. Genome Biol 4: R10. 